\title{
Replenishment policy for non-instantaneous deteriorating items in a two storage facilities under inflationary conditions
}

\author{
Chandra K. Jaggi*, Sunil Tiwari and Satish K. Goel
}

Department of Operational Research, Faculty of Mathematical Sciences, New Academic Block, University of Delhi, Delhi 110007, India

\begin{tabular}{l}
\hline C H R O N I C L E \\
\hline Article history: \\
Received August 312015 \\
Received in Revised Format \\
Septmber 26 2015 \\
Accepted December 22015 \\
Available online \\
December 2 2015 \\
\hline Keywords: \\
Inventory \\
Non-instantaneous deterioration \\
Two-warehouse \\
Partial backlogging \\
Inflation
\end{tabular}
\begin{abstract}
A B S T R A C T
The present study investigates an inventory model for non-instantaneous deteriorating items under inflationary conditions with partially backlogged shortages. In today's market structure consumers are looking for goods for which there is a delay in deterioration. At the same time, the consumers' willingness to wait has been decreased over time, which leads to lost sales. Moreover in financial decision-making, the effects of inflation and time value of money cannot be oblivious to an inventory system. In this scenario, managing inventory of goods remains a challenging task for the decision makers, who may also have to rent warehouse under different prevailing factors such as, bulk discount, limited space in the retail outlet, or increasing inflation rates. With a focus on reduction of costs and increasing customer service, warehouse decision models are crucial for an organization's profitability. Hence a mathematical model has been developed in the view of above scenario, in order to determine the optimal policy for the decision maker, by minimizing the present worth of total cost. The optimization procedure has been illustrated by a numerical example and detailed sensitivity analysis of the optimal solution has been performed to showcase the effect of various parameters. Managerial implications has also been presented to aid the decision making process.
\end{abstract}

C 2016 Growing Science Ltd. All rights reserved

\section{Introduction}

For every business one of the important questions is how to control and maintain the inventories of items. Often the demand is not anticipatory and thus to prevent the loss of customer base the firm needs to stock a higher inventory, which obviously requires additional storage capacity other than its owned warehouse $(\mathrm{OW})$. The additional storage space required by the organization to store the surplus inventory is called as rented warehouse (RW), which is assumed to be of abundant capacity. The RW is often equipped with the better preserving facilities that reduce the deterioration of goods in comparison to that of $\mathrm{OW}$ and hence result in higher holding cost than OW. An early discussion on the effect of two warehouse was considered by Hartely (1976) in which he assumed that the holding cost in rented warehouse (RW) is greater than that in own warehouse (OW), therefore, items in RW are first transferred to OW to meet the demand until the stock level in RW drops to zero and then items in OW are released. In recent years a lot of work has been done in the two-warehouse inventory system. One may refer to the studies of Zhou and Yang (2005), Lee

* Corresponding author. TelFax: 91-11-27666672

E-mail: ckjaggi@yahoo.com (C. K. Jaggi) 
(2006), Liang and Zhou (2011), Hsieh et al. (2008), Niu and Xie, (2008), Lee and Hsu (2009), Jaggi et al. (2011) Yang and Chang (2013), Chung and Huang (2007), Liang and Zhou (2011), Bhunia et al. (2014) and Jaggi et al. (2014).

Another phenomenon that affects the inventory systems is deterioration. Items in inventory are subjected to several risks such as pilferage, breakage, evaporation and obsolescence. Items such as food items, pharmaceuticals, chemicals, blood, gasoline, and radioactive chemicals, etc. deteriorate rapidly over time. Thus, the loss from deterioration needs to be regarded while deciding the economic order quantity. Ghare and Schrader (1963) presented an EOQ model for deteriorating items assuming exponential decay. Covert and Phillip (1973) extended Ghare and Schrader's (1963) model with the assumption of Weibull distribution deterioration. Further research in this area is summarized by different survey papers Raafat et al. (1991), Goyal and Giri (2001) and Bakker et al. (2012).

Models developed in all the above mentioned papers considered that the deterioration of items starts as soon as items arrive in the inventory. However, in reality there are some items which have a longer shelf life and start to perish only after some time (termed as non-instantaneous deteriorating items). This characteristic can be usually observed in almost all food stuffs, fashionable items, electronics products etc. Wu et al. (2006) introduced the phenomenon "non-instantaneous deterioration" and established the optimal replenishment policy for non-instantaneous deteriorating item with stock dependent demand and partial backlogging. Subsequently, many researchers such as Ouyang et al. (2006, 2008), Wu et al. (2009), Jaggi and Verma (2010), Chang et al. (2010), Geetha et al. (2010), Soni et al. (2012), Maihami and Kamalabadi (2012a, 2012b), Shah et al. (2013), Dye (2013) and Tsao (2014), have studied the inventory models for non-instantaneous deteriorating items under various conditions.

But, before making any investment, understanding of inflation and time value of money is crucial. Inflation brings price rise and decreases real value of money. To get the real estimate of all costs incurred, it is logical to incorporate the net effect of inflation and time value of money. Many authors have developed different inventory models under inflationary conditions with different assumptions. Buzacott (1975) and Misra (1975) were the first to elaborate the significance of inflation and time value of money by developing inventory models with constant inflation rate. Bierman and Thomas (1977) proposed the EOQ model considering the effect of both inflation and time value of money. Misra (1979) first continued the previous work for different inflation rates for various associated costs. Bose (1995) developed a model under inflation and time value of money using Discounted Cash Flow (DCF) approach. Further Jaggi et al. (1997) examined the effect of changing inflation rates and credit policies for non-deteriorating items. Thereafter, several interesting research papers have appeared e.g. Yang et al. (2001), Sarkar et al.(2000), Moon and Lee (2000), Wee and Law (2001), Yang (2004, 2006, 2012). Recently, great amount of effort has been put to develop models including different combinations of trade credit, imperfect items, inflation and time value of money e.g. Sarkar and Moon (2011) examined the effect of inflation on imperfect quantity items. Chung et al. (2001) formulated economic replenishment model under the consideration of time discounting. Jaggi et al. (2006) studied the effect of inflation-induced demand on order policies for deteriorating items. Later Jaggi and Khanna (2009) developed retailer's procurement policy under inflationary conditions when the end demand is credit-linked.

Incorporating all the above facts, this paper develops a two-warehouse inventory model for noninstantaneous deteriorating items when shortages are allowed and partially backlogged under inflationary condition and determines the optimal policies by minimizing the present worth of total cost. The optimization procedure has been illustrated by a numerical example and detailed sensitivity analysis of the optimal solution has been performed to showcase the effect of various parameters.

The rest of this paper is organized as follows. Section 2 defines the assumptions and notation used throughout the entire paper. Section 3 provides a formulation of the inventory model. Section 4 discusses the optimality of the objective function. Section 5 proposes an algorithm to find the optimal solutions. 
Special Cases are discussed in Section 6. Section 7 illustrates the proposed inventory model with numerical examples along-with Sensitivity analysis. Finally, conclusions are made and future research directions are outlined in Section 8.

\section{Assumption and Notations}

The mathematical models of the two-warehouse inventory problems are based on the following assumptions:

1. The own warehouse (OW) has a fixed capacity of $\mathrm{W}$ units; the rented warehouse (RW) has unlimited capacity.

2. The inventory costs (including holding cost and deterioration cost) in the RW are higher than those in OW.

3. Demand rate is assumed to be deterministic and constant.

4. Replenishment rate is instantaneous.

5. Lead-time is negligible.

6. The planning horizon of the inventory system is infinite.

7. Unsatisfied demand/shortages are allowed and partially backlogged. During the stock-out period, the backlogging rate is variable and is dependent on the length of the waiting time for next replenishment. So that the backlogging rate for the negative inventory is $e^{-\delta(T-t)}$; where $\delta(>0)$ denotes the backlogging parameter and $(T-t)$ is waiting time for the next replenishment.

In addition, the following notations are used throughout this paper.

A replenishment cost per order

$c \quad$ purchasing cost per unit

$A_{1} \quad$ replenishment cost per order in the single-warehouse system

$W \quad$ capacity of the owned warehouse

$D \quad$ demand rate per unit time

$Q \quad$ order quantity per cycle

$Z \quad$ maximum inventory level per cycle.

$H \quad$ holding cost per unit per unit time in OW.

$F \quad$ holding cost per unit per unit time in RW, where $F>H$

$s \quad$ the backlogging cost per unit per unit time, if shortage is backlogged.

$c_{1} \quad$ unit opportunity cost due to lost sale, if the shortage is lost.

$R \quad$ the inflation rate.

$\alpha \quad$ deterioration rate in $\mathrm{OW}$, where $0 \leq \alpha<1$

$\beta \quad$ deterioration rate in $\mathrm{RW}$, where $0 \leq \beta<1 ; \beta<\alpha$.

$t_{d} \quad$ time period during which no deterioration occurs.

$t_{r} \quad$ time at which the inventory level reaches zero in RW.

$t_{w} \quad$ time at which the inventory level reaches zero in $\mathrm{OW}$.

$T \quad$ the length of the replenishment cycle in year.

$I_{0}(t) \quad$ inventory level in the OW at any time $t$ where $0 \leq t \leq T$

$I_{r}(t) \quad$ inventory level in the RW at any time $t$ where $0 \leq t \leq T$

$T C_{i} \quad$ present value of total relevant cost per unit time for case $\mathrm{i}=1,2$

$B(t) \quad$ backlogged level at any time $t$ where $t_{w} \leq t \leq T$

$L(t) \quad$ number of lost sales at any time $t$ where $t_{w} \leq t \leq T$ 


\section{Model Formulation and Description}

This section formulates the replenishment problem of a two warehousing inventory model for a single non-instantaneous deteriorating item with complete backlogging. Initially, a lot size of $Q$ units enters in the system. After meeting the backorders, $Z$ units left in the inventory system, out of which $W$ units are kept in OW and the remaining $(Z-W)$ units are kept in the RW. As the deterioration of item is noninstantaneous, so initially, the units do not deteriorate for the time period $\left(t_{d}\right)$ and after that the deterioration begins. Which leads to two possible cases: when $t_{d}$ (time during which no deterioration occurs) is less than $t_{r}$ (time during which inventory in RW becomes zero) and when $t_{d}$ is greater than and equals to $t_{r}$.

\subsection{When $t_{d}<t_{r}$}

During the time interval $\left[0, t_{d}\right]$, there is no deterioration, the inventory in RW is depleted only due to demand whereas in OW inventory level remains the same. In the time interval $\left[t_{d}, t_{r}\right]$ the inventory level in RW is dropping to zero due to the combined effect of demand and deterioration and in OW inventory depleted due to deterioration only. Further, during the time interval $\left[t_{r}, t_{w}\right]$ depletion of inventory occurs in OW due to the combined effect of demand and deterioration and it reaches to zero at time $t_{w}$. Moreover, during the interval $\left[t_{w}, T\right]$, the demand is backlogged. The behavior of the model over the whole cycle $[0, T]$ has been graphically represented below in Fig. 1.

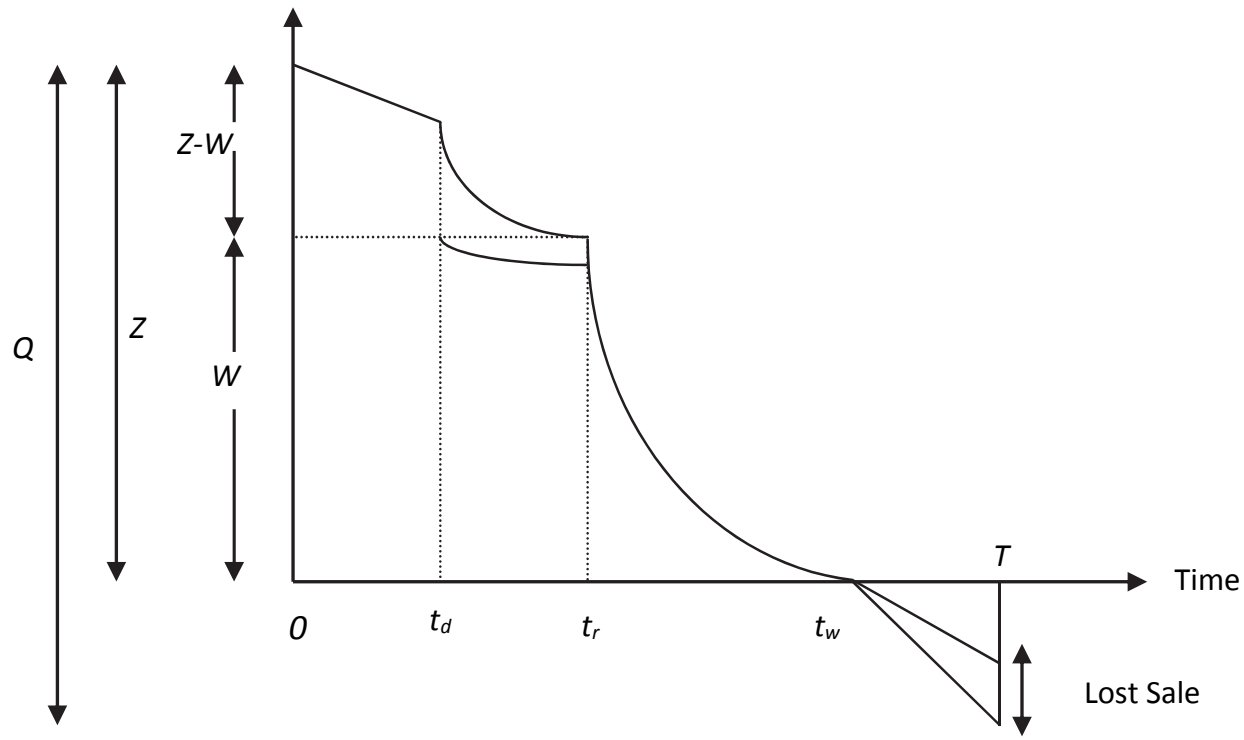

Fig. 1. Two-warehouse inventory system when $t_{d}<t_{r}$

Therefore, the differential equations that describe the inventory level in the RW and OW at any time $t$ over the period $(0, T)$ are given by:

$$
\begin{array}{ll}
\frac{d I_{r}(t)}{d t}=-D, & 0 \leq t \leq t_{d} \\
\frac{d I_{r}(t)}{d t}+\beta I_{r}(t)=-D, & t_{d}<t \leq t_{r}
\end{array}
$$




$$
\begin{array}{ll}
\frac{d I_{0}(t)}{d t}+\alpha I_{0}(t)=0, & t_{d}<t \leq t_{r} \\
\frac{d I_{0}(t)}{d t}+\alpha I_{0}(t)=-D, & t_{r}<t \leq t_{w} \\
\frac{d B(t)}{d t}=D e^{-\delta(T-t)}, & t_{w}<t \leq T
\end{array}
$$

The solutions of the above five differential Eqs.(1-5) with boundary conditions $I_{r}(0)=Z-W, I_{r}\left(t_{r}\right)=0, I_{0}\left(t_{d}\right)=W, I_{0}\left(t_{w}\right)=0 \& B\left(t_{w}\right)=0$ respectively are

$$
\begin{array}{rlrl}
I_{r}(t) & =Z-D t-W, & & 0 \leq t \leq t_{d} \\
I_{r}(t) & =\frac{D}{\beta}\left(e^{\beta\left(t_{r}-t\right)}-1\right), & t_{d}<t \leq t_{r} \\
I_{0}(t)=W e^{\alpha\left(t_{d}-t\right)}, & t_{d}<t \leq t_{r} \\
I_{0}(t)=\frac{D}{\alpha}\left(e^{\alpha\left(t_{w}-t\right)}-1\right), & t_{r}<t \leq t_{w} \\
B(t)=\frac{D}{\delta}\left\{e^{-\delta(T-t)}-e^{-\delta\left(T-t_{w}\right)}\right\}, t_{w}<t \leq T
\end{array}
$$

The number of lost sales at time $t$ is $L(t)=\int_{t_{w}}^{t} D\left\{1-e^{-\delta(T-t)}\right\} d t ; \quad t_{w} \leq t \leq T$

$$
=D\left[\left(t-t_{w}\right)-\frac{1}{\delta}\left\{e^{-\delta(T-t)}-e^{-\delta\left(T-t_{w}\right)}\right\}\right]
$$

Considering continuity of $I_{r}(t)$ at $t=t_{d}$, it follows from Eq. (6) and Eq. (7) that

$$
Z-D t_{d}-W=\frac{D}{\beta}\left(e^{\beta\left(t_{r}-t_{d}\right)}-1\right),
$$

which implies that the maximum inventory level per cycle is

$$
Z=W+D t_{d}+\frac{D}{\beta}\left(e^{\beta\left(t_{r}-t_{d}\right)}-1\right)
$$

Considering continuity of $I_{0}(t)$ at $t=t_{r}$ it follows from Eq. (8) and Eq. (9) that

$$
\begin{aligned}
& W e^{\alpha\left(t_{d}-t_{r}\right)}=\frac{D}{\alpha}\left(e^{\alpha\left(t_{w}-t_{r}\right)}-1\right), \\
& t_{w}=t_{r}+\frac{1}{\alpha} \ln \left(\frac{D+\alpha W e^{\alpha\left(t_{d}-t_{r}\right)}}{D}\right) .
\end{aligned}
$$

Putting $t=T$ in Eq. (10), the maximum amount of demand backlogged per cycle is

$$
B(T)=\frac{D}{\delta}\left(1-e^{-\delta\left(T-t_{w}\right)}\right)
$$

Therefore, the order quantity over the replenishment cycle can be determined as $Q=Z+B(T)$ (Using Eq. (13) and Eq. (15)) 


$$
=W+D t_{d}+\frac{D}{\beta}\left(e^{\beta\left(t_{r}-t_{d}\right)}-1\right)+\frac{D}{\delta}\left(1-e^{-\delta\left(T-t_{w}\right)}\right)
$$

The total cost per cycle consists of the following elements:

1. Present worth of the replenishment cost is $A$

2. Present worth of the inventory holding cost in RW

$$
\begin{aligned}
=F & \left(\int_{0}^{t_{d}} e^{-R t} I_{r}(t) d t+\int_{t_{d}}^{t_{r}} e^{-R t} I_{r}(t) d t\right) \\
=F & {\left[\frac{Z-W}{R}-\frac{\left(Z-W-D t_{d}\right) e^{-R t_{d}}}{R}+\frac{D}{R^{2}}\left\{e^{-R t_{d}}-1\right\}+\frac{D}{\beta}\left\{\frac{1}{\beta+R}\left\{e^{\beta t_{r}-(\beta+R) t_{d}}-e^{-R t_{r}}\right\}\right.\right.} \\
& \left.\left.+\frac{1}{R}\left\{e^{-R t_{r}}-e^{-R t_{d}}\right\}\right\}\right]
\end{aligned}
$$

3. Present worth of the inventory holding cost in OW

$$
\begin{aligned}
& =H\left(\int_{0}^{t_{d}} W e^{-R t} d t+\int_{t_{d}}^{t_{r}} e^{-R t} I_{0}(t) d t+\int_{t_{r}}^{t_{W}} e^{-R t} I_{0}(t) d t\right) \\
& =H\left[\frac{W}{R}\left\{1-e^{-R t_{d}}\right\}+\frac{D}{R+\alpha}\left\{e^{-R t_{d}}-e^{\alpha t_{d}-(R+\alpha) t_{r}}\right\}+\frac{D}{\alpha}\left\{\frac{1}{R+\alpha}\left\{e^{\alpha t_{w}-(R+\alpha) t_{r}}-e^{-R t_{w}}\right\}\right.\right. \\
& \left.\left.+\frac{1}{R}\left\{e^{-R t_{w}}-e^{-R t_{r}}\right\}\right\}\right]
\end{aligned}
$$

4. Present worth of the backlogging cost is $s \int_{t_{W}}^{T} B(t) e^{-R t} d t$

$$
=\frac{s D}{\delta} e^{-\delta T}\left[\frac{1}{\delta-R}\left\{e^{(\delta-R) T}-e^{(\delta-R) t_{w}}\right\}+\frac{e^{\delta t_{w}}}{R}\left\{e^{-R T}-e^{-R t_{w}}\right\}\right]
$$

5. Present worth of the opportunity cost due to lost sale is

$$
=c_{1} e^{-R T} \int_{t_{w}}^{T}\left\{1-e^{-\delta(T-t)}\right\} D d t
$$

6. Present worth of the Deterioration cost is

$$
\begin{gathered}
=c\left[\beta \int_{t_{d}}^{t_{r}} I_{r}(t) e^{-R t} d t+\alpha \int_{t_{d}}^{t_{r}} e^{-R t} I_{0}(t) d t+\alpha \int_{t_{r}}^{t_{w}} e^{-R t} I_{0}(t) d t\right] \\
=c D\left[\frac{1}{\beta+R}\left\{e^{\beta t_{r}-(\beta+R) t_{d}}-e^{-R t_{r}}\right\}+\frac{\alpha}{R+\alpha}\left\{e^{-R t_{d}}-e^{\alpha t_{d}-(R+\alpha) t_{r}}\right\}+\frac{1}{\alpha+R}\left\{e^{\alpha t_{w}-(\alpha+R) t_{r}}-e^{-R t_{w}}\right\}+\frac{1}{R}\left\{e^{-R t_{w}}-e^{-R t_{r}}\right\}\right]
\end{gathered}
$$

\subsection{When $t_{d} \geq t_{r}$}

In this case, time during which no deterioration occurs is greater than and equal to the time during which inventory in RW becomes zero and the behavior of the model over the whole cycle $[0, T]$ has been graphically represented as in Fig. 2 


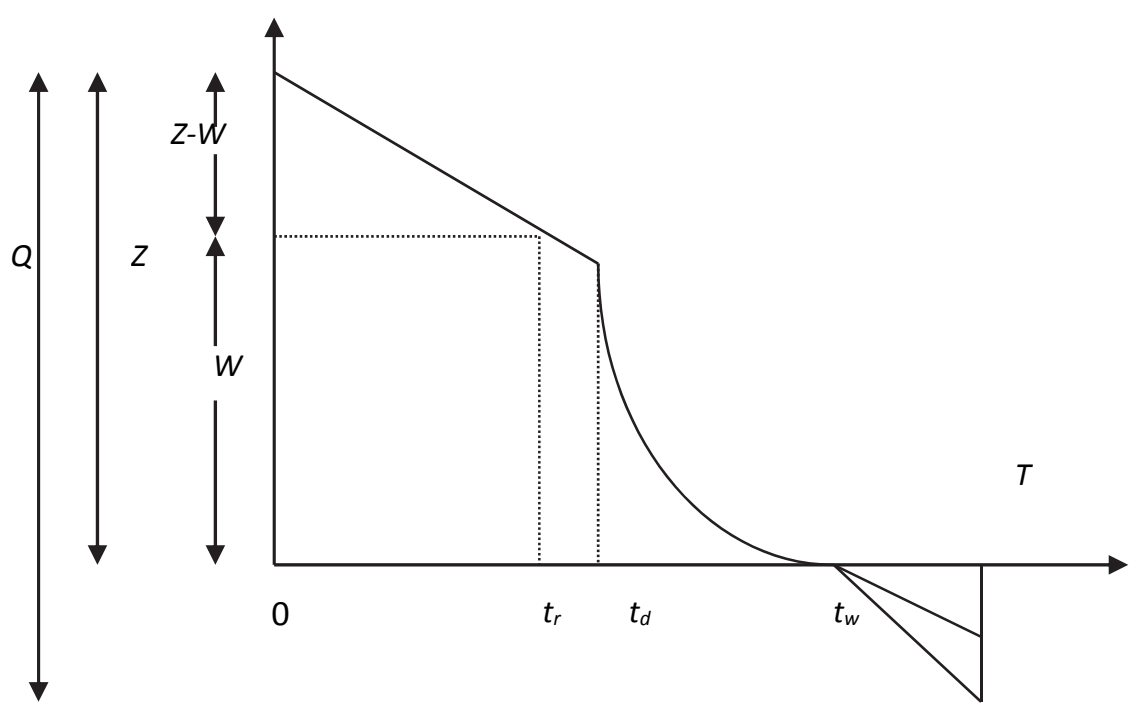

Fig. 2. Two-warehouse inventory system when $t_{d} \geq t_{r}$

The differential equations that describe the inventory level in the RW and OW at any time $t$ over the period $(0, T)$ are given by:

$$
\begin{array}{ll}
\frac{d I_{r}(t)}{d t}=-D, & 0 \leq t \leq t_{r} \\
\frac{d I_{0}(t)}{d t}=-D, & t_{r}<t \leq t_{d} \\
\frac{d I_{0}(t)}{d t}+\alpha I_{0}(t)=-D, & t_{d}<t \leq t_{w} \\
\frac{d B(t)}{d t}=D e^{-\delta(T-t)}, & t_{w}<t \leq T
\end{array}
$$

The solutions of the above four differential Eqs. (17-20) with boundary conditions $I_{r}\left(t_{r}\right)=0, I_{0}\left(t_{r}\right)=W, I_{0}\left(t_{W}\right)=0, B\left(t_{W}\right)=0$ respectively are

$$
\begin{array}{rlrl}
I_{r}(t) & =D\left(t_{r}-t\right), & & \leq t \leq t_{r} \\
I_{0}(t) & =W+D t_{r}-D t, & t_{r} & <t \leq t_{d} \\
I_{0}(t) & =\frac{D}{\alpha}\left(e^{\alpha\left(t_{w}-t\right)}-1\right), & t_{d} & <t \leq t_{w} \\
B(t) & =\frac{D}{\delta}\left\{e^{-\delta(T-t)}-e^{-\delta\left(T-t_{w}\right)}\right\}, t_{w} & <t \leq T
\end{array}
$$

The number of lost sales at time $t$ is

$$
\begin{aligned}
L(t) & =\int_{t_{w}}^{t} D\left\{1-e^{-\delta(T-t)}\right\} d t ; \quad t_{w} \leq t \leq T \\
& =D\left[\left(t-t_{w}\right)-\frac{1}{\delta}\left\{e^{-\delta(T-t)}-e^{-\delta\left(T-t_{w}\right)}\right\}\right]
\end{aligned}
$$

Considering continuity of $I_{0}(t)$ at $t=t_{d}$, it follows from Eq. (22) and Eq. (23) that 


$$
\begin{aligned}
& W+D t_{r}-D t_{d}=\frac{D}{\alpha}\left(e^{\alpha\left(t_{w}-t_{d}\right)}-1\right), \\
& t_{w}=t_{d}+\frac{1}{\alpha} \ln \left|1+\frac{\alpha W}{D}+\alpha\left(t_{r}-t_{d}\right)\right| .
\end{aligned}
$$

Now, at $t=0$ when $I_{r}(t)=Z-W$ and solving Eq. (21) we get the maximum inventory as

$$
Z=W+D t_{r}
$$

Putting $t=T$ in Eq. (24), the maximum amount of demand backlogged per cycle is

$$
B(T)=\frac{D}{\delta}\left(1-e^{-\delta\left(T-t_{w}\right)}\right) .
$$

Therefore, order quantity is $Q=Z+B(T)$

$$
=W+D t_{r}+\frac{D}{\delta}\left(1-e^{-\delta\left(T-t_{w}\right)}\right)(\operatorname{Using} \text { Eqs }(28-29))
$$

Again, the total cost per cycle consists of the following elements:

1. Present worth of the replenishment cost $=A$

2. Present worth of the inventory holding cost in $\mathrm{RW}=F \int_{0}^{t_{r}} e^{-R t} I_{r}(t) d t=\frac{F D}{R}\left[t_{r}+\frac{1}{R}\left\{e^{-R t_{r}}-1\right\}\right]$

3. Present worth of the inventory holding cost in OW

$$
\begin{gathered}
=H\left(\int_{0}^{t_{r}} e^{-R t} W d t+\int_{t_{r}}^{t_{d}} e^{-R t} I_{0}(t) d t+\int_{t_{d}}^{t_{w}} e^{-R t} I_{0}(t) d t\right) \\
=H\left[\frac{W}{R}-\left\{W+D\left(t_{r}-t_{d}\right)\right\} \frac{e^{-R t_{d}}}{R}+\frac{D}{\alpha}\left\{\frac{1}{\alpha+R}\left\{e^{\alpha t_{w}-(\alpha+R) t_{d}}-e^{-R t_{w}}\right\}+\frac{1}{R}\left\{e^{-R t_{w}}-e^{-R t_{d}}\right\}\right\}\right]
\end{gathered}
$$

4. Present worth of the backlogging cost $=s \int_{t_{W}}^{T} B(t) e^{-R t} d t$

$$
=\frac{s D}{\delta} e^{-\delta T}\left[\frac{1}{\delta-R}\left\{e^{(\delta-R) T}-e^{(\delta-R) t_{w}}\right\}+\frac{e^{\delta t_{w}}}{R}\left\{e^{-R T}-e^{-R t_{w}}\right\}\right]
$$

5. Present worth of opportunity cost due to lost sale $=c_{1} e^{-R T} \int_{t_{w}}^{T}\left\{1-e^{-\delta(T-t)}\right\} D d t$

$$
=c_{1} D e^{-R T}\left[T-t_{w}-\frac{1}{\delta}\left\{1-e^{-\delta\left(T-t_{w}\right)}\right\}\right]
$$

6. Present worth of the cost for deteriorated items $=c_{1} \alpha \int_{t_{d}}^{t_{w}} e^{-R t} I_{0}(t) d t$

$$
=c D\left[\frac{1}{R+\alpha}\left\{e^{\alpha t_{w}-(R+\alpha) t_{d}}-e^{-R t_{w}}\right\}+\frac{1}{R}\left\{e^{-R t_{w}}-e^{-R t_{d}}\right\}\right]
$$

Therefore, combining the above-mentioned two cases, the present worth of the total relevant cost per unit time during the cycle $(0, T)$ is given by

$$
T C\left(t_{r}, T\right)=\left\{\begin{array}{lll}
T C_{1}\left(t_{r}, T\right) & \text { if } & t_{d}<t_{r} \\
T C_{2}\left(t_{r}, T\right) & \text { if } & t_{d} \geq t_{r}
\end{array}\right.
$$


which is a function of two continuous variable $t_{r}$ and $T$.

where

$$
\begin{aligned}
& T C_{1}\left(t_{r}, T\right)=\frac{1}{T}\left[A+F\left\{\frac{Z-W}{R}-\frac{\left(Z-W-D t_{d}\right) e^{-R t_{d}}}{R}+\frac{D}{R^{2}}\left\{e^{-R_{t_{d}}}-1\right\}+\frac{D}{\beta}\left\{\frac{1}{\beta+R}\left\{e^{\beta t_{r}-(\beta+R) t_{d}}-e^{-R t_{r}}\right\}+\frac{1}{R}\left\{e^{-R t_{r}}-e^{-R t_{d}}\right\}\right\}\right\}\right. \\
& +H\left\{\frac{W}{R}\left\{1-e^{-R t_{d}}\right\}+\frac{D}{R+\alpha}\left\{e^{-R t_{d}}-e^{\alpha t_{d}-(R+\alpha) t_{r}}\right\}+\frac{D}{\alpha}\left\{\frac{1}{R+\alpha}\left\{e^{\alpha t_{w}-(R+\alpha) t_{r}}-e^{-R t_{w}}\right\}+\frac{1}{R}\left\{e^{-R t_{w}}-e^{-R t_{r}}\right\}\right\}\right\} \\
& +\frac{s D}{\delta} e^{-\delta T}\left\{\frac{1}{\delta-R}\left\{e^{(\delta-R) T}-e^{(\delta-R) t_{w}}\right\}+\frac{e^{\delta t_{w}}}{R}\left\{e^{-R T}-e^{-R t_{w}}\right\}\right\}+c_{1} D e^{-R T}\left\{T-t_{w}-\frac{1}{\delta}\left\{1-e^{-\delta\left(T-t_{w}\right)}\right\}\right\} \\
& \left.+c D\left\{\frac{1}{\beta+R}\left\{e^{\beta t_{r}-(\beta+R) t_{d}}-e^{-R_{t_{r}}}\right\}+\frac{\alpha}{R+\alpha}\left\{e^{-R_{t_{d}}}-e^{\alpha t_{d}-(R+\alpha) t_{r}}\right\}+\frac{1}{\alpha+R}\left\{e^{\alpha t_{w}-(\alpha+R) t_{r}}-e^{-R t_{w}}\right\}+\frac{1}{R}\left\{e^{-R_{t_{w}}}-e^{-R t_{r}}\right\}\right\}\right] \\
& T C_{2}\left(t_{r}, T\right)=\frac{1}{T}\left[A+\frac{F D}{R}\left\{t_{r}+\frac{1}{R}\left\{e^{-R t_{r}}-1\right\}\right\}+H\left\{\frac{W}{R}-\left\{W+D\left(t_{r}-t_{d}\right)\right\} \frac{e^{-R t_{d}}}{R}+\frac{D}{\alpha}\left\{\frac{1}{\alpha+R}\left\{e^{\alpha t_{w}-(\alpha+R) t_{d}}-e^{-R_{t_{w}}}\right\}\right.\right.\right. \\
& \left.\left.+\frac{1}{R}\left\{e^{-R t_{w}}-e^{-R t_{d}}\right\}\right\}\right\}+\frac{s D}{\delta} e^{-\delta T}\left\{\frac{1}{\delta-R}\left\{e^{(\delta-R) t_{w}}+e^{(\delta-R) T}\right\}-\frac{e^{\delta t_{w}}}{R}\left\{e^{-R T}-e^{-R t_{w}}\right\}\right\} \\
& \left.+c_{1} D e^{-R T}\left\{T-t_{w}-\frac{1}{\delta}\left\{1-e^{-\delta\left(T-t_{w}\right)}\right\}\right\}+c D\left\{\frac{1}{R+\alpha}\left\{e^{\alpha t_{w}-(R+\alpha) t_{d}}-e^{-R t_{w}}\right\}+\frac{1}{R}\left\{e^{-R t_{w}}-e^{-R t_{d}}\right\}\right\}\right]
\end{aligned}
$$

\section{Convexity of the Cost function}

In this section, we discuss the convexity of the objective function. The necessary conditions for the total cost to be minimum is

$$
\frac{\partial T C_{i}\left(t_{r}, T\right)}{\partial t_{r}}=0, \text { and } \frac{\partial T C_{i}\left(t_{r}, T\right)}{\partial T}=0 \quad \text { for } i=1,2 \text { which gives }
$$

which gives the optimal values of $t_{r i}$ and $T_{i}$ (say $t_{r i}{ }^{*}$ and $T_{i}{ }^{*}$ ) for $i=1,2$ provided, it also satisfies the following sufficient conditions.

The sufficient conditions for minimizing $T C_{i}\left(t_{r}, T\right)$ using the Hessian matrix $H$, which is a the matrix of second order partial derivatives are

$$
\begin{gathered}
H=\left[\begin{array}{ll}
\frac{\partial^{2} T C_{i}\left(t_{r}, T\right)}{\partial t_{r}^{2}} & \frac{\partial^{2} T C_{i}\left(t_{r}, T\right)}{\partial t_{r} \partial T} \\
\frac{\partial^{2} T C_{i}\left(t_{r}, T\right)}{\partial T \partial t_{r}} & \frac{\partial^{2} T C_{i}\left(t_{r}, T\right)}{\partial T^{2}}
\end{array}\right] \\
D_{1}=\frac{\partial^{2} T C_{i}\left(t_{r}, T\right)}{\partial t_{r}^{2}}>0, \text { and } D_{2}=\operatorname{det} H=\left|\begin{array}{ll}
\frac{\partial^{2} T C_{i}\left(t_{r}, T\right)}{\partial t_{r}^{2}} & \frac{\partial^{2} T C_{i}\left(t_{r}, T\right)}{\partial t_{r} \partial T} \\
\frac{\partial^{2} T C_{i}\left(t_{r}, T\right)}{\partial T \partial t_{r}} & \frac{\partial^{2} T C_{i}\left(t_{r}, T\right)}{\partial T^{2}}
\end{array}\right|>0 \text { for } i=1 \text { and } 2 .
\end{gathered}
$$

Here $D_{1}$ and $D_{2}$ are minors of the Hessian matrix $H$.

The first and second order derivatives have been calculated and these are very complicated (Appendix 1

- 2). Therefore, it is very difficult to prove the convexity mathematically; however the convexity of all 
the total cost functions has been established graphically (figures 3 - 4) with the help of MATLAB. Hence our required optimal solution is a global one.

\section{Solution Procedure}

The procedure for finding the economic ordering policy is as follows:

Step 1: Determine $t_{r l}{ }^{*}$ and $T_{1}{ }^{*}$ from Eq. (32). If $t_{d}<t_{r}$, substituting these values in the Eq. (13), Eq. (16) and Eq. (31.a), the optimal values of $Z^{*}, Q^{*} \& T C_{1}\left(t_{r}{ }^{*}, T^{*}\right)$ can be obtained respectively, else $T C_{1}$ $\left(t_{r}{ }^{*}, T^{*}\right)=\infty$.

Step 2: Determine $t_{r 2}{ }^{*}$ and $T_{2}{ }^{*}$ from Eq. (32). If $t_{d} \geq t_{r}$ substituting these values in the Eq. (28), Eq. (30) and Eq. (31.b), the optimal values of $Z^{*}, Q^{*} \& T C_{2}\left(t_{r}{ }^{*}, T^{*}\right)$ can be obtained respectively, else $T C_{2}$ $\left(t_{r}{ }^{*}, T^{*}\right)=\infty$.

Step 3: Now compare the total optimal cost for both cases i.e. $T C_{1}\left(t_{r}{ }^{*}, T^{*}\right)$ and $T C_{2}\left(t_{r}{ }^{*}, T^{*}\right)$. The policy having minimum total optimal cost is optimal one.

\section{Some Special cases}

\subsection{When $W=\infty$ i.e. Single Warehouse Case}

In this case, the present worth of total cost per unit time given by Eq. (31) reduces to

$$
T C\left(t_{w}, T\right)=\left\{\begin{array}{lll}
T C_{3}\left(t_{w}, T\right) & \text { if } & t_{d}<t_{w} \\
T C_{4}\left(t_{w}, T\right) & \text { if } & t_{d} \geq t_{w}
\end{array}\right.
$$

where

$$
\begin{aligned}
T C_{3}\left(t_{w}, T\right)= & \frac{1}{T}\left[A_{1}+H\left\{\frac{1}{R}\left\{Z-\left(Z-D t_{d}\right) e^{-R t_{d}}\right\}+\frac{D}{R^{2}}\left(e^{-R t_{d}}-1\right)+\frac{D}{\alpha}\left\{\frac{1}{R+\alpha}\left\{e^{\alpha t_{w}-(R+\alpha) t_{d}}-e^{-R t_{w}}\right\}+\frac{1}{R}\left\{e^{-R t_{w}}-e^{-R t_{d}}\right\}\right\}\right.\right. \\
& +\frac{s D}{\delta} e^{-\delta T}\left\{\frac{1}{\delta-R}\left\{e^{(\delta-R) T}-e^{(\delta-R) t_{w}}\right\}+\frac{e^{\delta t_{w}}}{R}\left\{e^{-R T}-e^{-R t_{w}}\right\}\right\}+c_{1} D e^{-R T}\left\{T-t_{w}-\frac{1}{\delta}\left\{1-e^{-\delta\left(T-t_{w}\right)}\right\}\right\} \\
& \left.+c D\left\{\frac{1}{R+\alpha}\left\{e^{\alpha t_{w}-(R+\alpha) t_{d}}-e^{-R t_{w}}\right\}+\frac{1}{R}\left\{e^{-R t_{w}}-e^{-R t_{d}}\right\}\right\}\right]
\end{aligned}
$$

and

$$
\begin{aligned}
T C_{4}\left(t_{w}, T\right)=\frac{1}{T}[ & A_{1}+H\left\{\frac{1}{R}\left(1-e^{-R t_{w}}\right)\left(Z-\frac{D}{R}\right)+\frac{D}{R} t_{w} e^{-R t_{w}}+\frac{s D}{\delta} e^{-\delta T}\left\{\frac{1}{\delta-R}\left\{e^{(\delta-R) T}-e^{(\delta-R) t_{w}}\right\}+\frac{e^{\delta t_{w}}}{R}\left\{e^{-R T}-e^{-R t_{w}}\right\}\right\}\right. \\
& \left.+c_{1} D e^{-R T}\left\{T-t_{w}-\frac{1}{\delta}\left\{1-e^{-\delta\left(T-t_{w}\right)}\right\}\right\}\right]
\end{aligned}
$$

\subsection{When no Shortages are allowed}

The present worth of total cost per unit time given by Eq. (31) reduces to

$$
T C\left(t_{r}, T\right)=\left\{\begin{array}{lll}
T C_{5}\left(t_{r}, T\right) & \text { if } & t_{d}<t_{r} \\
T C_{6}\left(t_{r}, T\right) & \text { if } & t_{d} \geq t_{r}
\end{array}\right.
$$

where

$$
\begin{aligned}
T C_{5}\left(t_{r}, T\right)= & \frac{1}{T}\left[A+F\left\{\frac{Z-W}{R} \frac{\left(Z-W-D t_{d}\right) e^{-R_{d}}}{R}+\frac{D}{R^{2}}\left\{e^{-R_{t_{d}}}-1\right\}+\frac{D}{\beta}\left\{\frac{1}{\beta+R}\left\{e^{\beta t_{r}-(\beta+R) t_{d}}-e^{-R_{r}}\right\}+\frac{1}{R}\left\{e^{-R_{t_{r}}}-e^{-R_{d}}\right\}\right\}\right\}\right. \\
& +H\left\{\frac{W}{R}\left\{1-e^{-R_{d}}\right\}+\frac{D}{R+\alpha}\left\{e^{-R_{t_{d}}}-e^{t_{d}-(R+\alpha) t_{r}}\right\}+\frac{D}{\alpha}\left\{\frac{1}{R+\alpha}\left\{e^{\alpha T-(R+\alpha) t_{r}}-e^{-R T}\right\}+\frac{1}{R}\left\{e^{-R T}-e^{-R_{r}}\right\}\right\}\right\} \\
& \left.+C D\left\{\frac{1}{\beta+R}\left\{e^{\beta \beta_{r}-(\beta+R) t_{d}}-e^{-R_{r}}\right\}+\frac{1}{\alpha+R}\left\{e^{\alpha T-(\alpha+R) t_{r}}-e^{-R T}\right\}+\frac{1}{R}\left\{e^{-R T}-e^{-R_{d}}\right\}\right\}\right]
\end{aligned}
$$


and

$$
\begin{aligned}
T C_{6}\left(t_{r}, T\right)= & \frac{1}{T}\left[A+\frac{F D}{R}\left\{t_{r}+\frac{1}{R}\left\{e^{-R t_{r}}-1\right\}\right\}+H\left\{\frac{W}{R}-\left\{W+D\left(t_{r}-t_{d}\right)\right\} \frac{e^{-R t_{d}}}{R}\right.\right. \\
+ & \left.\frac{D}{\alpha}\left\{\frac{1}{\alpha+R}\left\{e^{\alpha T-(\alpha+R) t_{d}}-e^{-R T}\right\}+\frac{1}{R}\left\{e^{-R T}-e^{-R t_{d}}\right\}\right\}\right\} \\
& \left.+c D\left\{\frac{1}{R+\alpha}\left\{e^{\alpha T-(R+\alpha) t_{d}}-e^{-R T}\right\}+\frac{1}{R}\left\{e^{-R T}-e^{-R t_{d}}\right\}\right\}\right]
\end{aligned}
$$

\subsection{When $t_{d}=0$ i.e. instantaneous deterioration}

Here, the present worth of total cost per unit time given by Eq. (31) reduces to

$$
\begin{aligned}
& T C_{7}\left(t_{r}, T\right)=\frac{1}{T}\left[A++\frac{F D}{\beta}\left\{\frac{1}{R+\beta}\left\{e^{\beta_{r}}-e^{-R_{r}}\right\}+\frac{1}{R}\left\{e^{-R_{r_{r}}}-1\right\}\right\}+H\left\{\frac{W}{R+\alpha}\left\{1-e^{-(R+\alpha) t_{r}}\right\}+\frac{D}{\alpha}\left\{\frac{1}{R+\alpha}\left\{e^{\left.\alpha_{w}-(R+\alpha)\right)_{r}}-e^{-R_{w_{w}}}\right\}\right.\right.\right. \\
& \left.+\frac{1}{R}\left\{e^{-R_{t_{w}}}-e^{-R t_{r}}\right\}\right\}+\frac{s D}{\delta}\left\{\frac{1}{R-\delta}\left\{e^{-\delta T-(R-\delta) t_{w}}-e^{-R T}\right\}+\frac{1}{R} e^{-\delta\left(T-t_{w}\right)}\left\{e^{-R T}-e^{-R t_{w}}\right\}\right\}+c_{1} D e^{-R T}\left\{T-t_{w}-\frac{1}{\delta}\left\{1-e^{-\delta\left(T-t_{w}\right)}\right\}\right\} \\
& \left.+c\left\{F D\left\{\frac{1}{R+\beta}\left\{e^{\beta t_{r}}-e^{-R_{r}}\right\}+\frac{1}{R}\left\{e^{-R_{t_{r}}}-1\right\}\right\}+H\left\{\frac{W}{R+\alpha}\left\{1-e^{-(R+\alpha) t_{r}}\right\}+\frac{D}{\alpha}\left\{\frac{1}{R+\alpha}\left\{e^{a_{w}-(R+\alpha) t_{r}}-e^{-R_{w_{w}}}\right\}+\frac{1}{R}\left\{e^{-R_{t_{w}}}-e^{-R_{t_{r}}}\right\}\right\}\right\}\right\}\right]
\end{aligned}
$$

6.4. When there is no inflation and shortages are fully backlogged

Here, the present worth of total cost per unit time given by Eq. (31) reduces to

$$
\begin{aligned}
& T C\left(t_{r}, T\right)=\left\{\begin{array}{lll}
T C_{8}\left(t_{r}, T\right) & \text { if } & t_{d}<t_{r} \\
T C_{9}\left(t_{r}, T\right) & \text { if } & t_{d} \geq t_{r}
\end{array}\right. \\
& \text { where }^{T C_{8}\left(t_{r}, T\right)}=\frac{1}{T}\left[A+F\left\{Z t_{d}-\frac{D t_{d}^{2}}{2}-W t_{d}-\frac{D}{\beta}\left(t_{r}-t_{d}+\frac{1}{\beta}-\frac{e^{\beta\left(t_{r}-t_{d}\right)}}{\beta}\right)\right\}+H\left\{W t_{d}+\frac{W}{\alpha}\left(1-e^{\alpha\left(t_{d}-t_{r}\right)}\right)+\frac{D}{\alpha}\left(t_{r}-t_{w}-\frac{1}{\alpha}+\frac{e^{\alpha\left(t_{w}-t_{r}\right)}}{\alpha}\right)\right\}\right. \\
& \left.+s D\left(T-t_{w}\right)^{2} / 2+c(Q-D T)\right]
\end{aligned}
$$

and

$$
\begin{aligned}
T C_{9}\left(t_{r}, T\right)=\frac{1}{T}[ & A+\frac{F D t_{r}^{2}}{2}+H\left\{W t_{r}+D t_{r}\left(t_{d}-t_{r}\right)-\frac{D}{2}\left(t_{d}^{2}-t_{r}^{2}\right)+\frac{D}{\alpha}\left\{\frac{1}{\alpha}\left(e^{\alpha\left(t_{w}-t_{d}\right)}-1\right)-\left(t_{w}-t_{d}\right)\right\}\right\} \\
& \left.+s D\left(T-t_{w}\right)^{2} / 2+c(Q-D T)\right]
\end{aligned}
$$

which is same cost function as obtained by Jaggi and Verma (2010).

\section{Numerical Examples and Sensitivity analysis}

In this section, we develop a numerical analysis to validate the model developed.

Example 1: Let us consider an inventory system with the following data: $A=\$ 250 /$ order, $D=$ 300unit/year, $c=\$ 10 /$ unit, $p=\$ 15 /$ unit, $H=\$ 0.5 /$ unit/year, $F=\$ 0.7 /$ unit/year, $W=200$ units, $c_{1}=$ $\$ 5 /$ unit/year, $R=0.06, t_{d}=0.2, \alpha=0.05 /$ unit, $\beta=0.03 /$ unit.

Using the proposed solution procedure, the results are as follows, the optimal length of positive inventory $\left(t_{r}\right)$ is 0.903 year, $t_{w}$ is 1.18 year, the optimal cycle length $(T)$ is 1.34 year, the optimal order quantity $(Q)$ is 582.36 units, and the present worth of total optimal cost (TC) is $\$ 387.71$. 

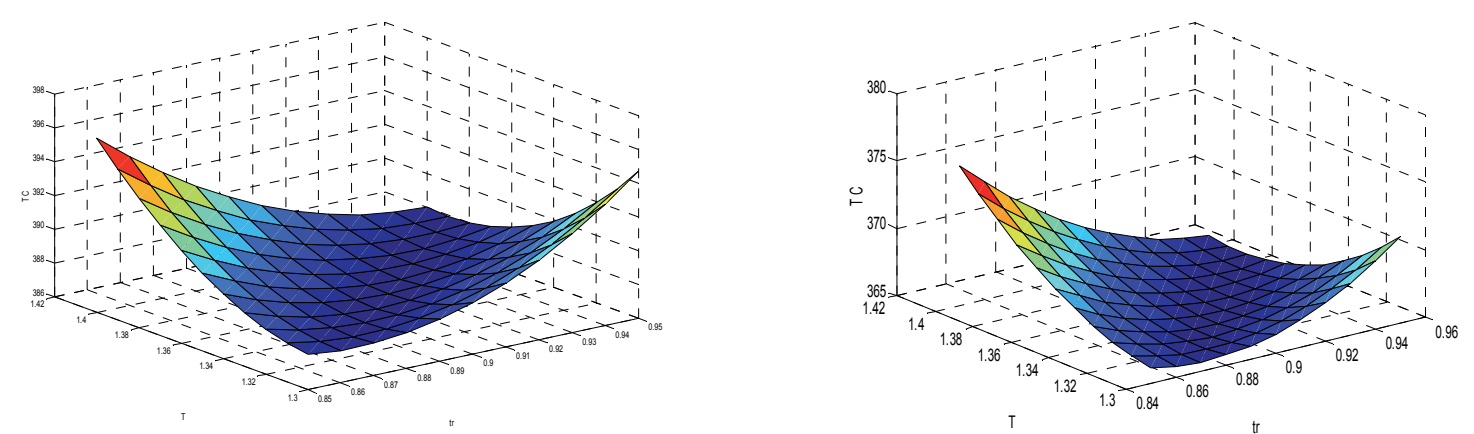

Fig. 3. Convexity of total cost function for example 1 Fig. 4. Convexity of total cost function for example 2

Example 2: Let us consider an inventory system with the following data: $A=\$ 250$ /order, $D=$ 300unit/year, $c=\$ 10 /$ unit, $p=\$ 15 /$ unit, $H=\$ 0.5 /$ unit/year, $F=\$ 0.7 /$ unit/year, $W=200$ units, $c 1=$ $\$ 5 /$ unit/year, $R=0.06, t_{d}=0.5, \alpha=0.05 /$ unit, $\beta=0.03 /$ unit.

Using the proposed solution procedure, the results are as follows, the optimal length of positive inventory $\left(t_{r}\right)$ is 0.49 year, $t_{w}$ is 1.03 year, the optimal cycle length $(T)$ is $1.15 y$ ear, the optimal order quantity $(Q)$ is 532.03 units, and the total optimal profit $(T P)$ is $\$ 338.03$. Further, the sensitivity analysis of optimal solution w.r.t. major parameters inflation $(\boldsymbol{R})$ and deterioration $(\boldsymbol{\alpha}$ and $\boldsymbol{\beta})$, non deteriorating period $\left(\boldsymbol{t}_{\boldsymbol{d}}\right)$ and backlogging rate $(\boldsymbol{\delta})$ has been shown in Tables $1-3$, respectively.

Table 1

Impact of inflation $(R)$ and deterioration on the optimal replenishment policy

\begin{tabular}{ccccccc}
\hline \multirow{2}{*}{$R$} & $\begin{array}{c}\text { Change in } \\
\text { Deterioration }\end{array}$ & $t_{r}$ & $t_{w}$ & $T$ & $Q$ & $\begin{array}{c}\text { Present Worth of Total } \\
\text { Optimal Cost }\end{array}$ \\
\hline \multirow{3}{*}{0.02} & $\alpha<\beta$ & 0.86 & 1.14 & 1.31 & 571.23 & 398.74 \\
& $\alpha=\beta$ & 0.87 & 1.15 & 1.31 & 572.44 & 400.09 \\
\hline \multirow{3}{*}{0.04} & $\alpha>\beta$ & 0.88 & 1.16 & 1.32 & 573.66 & 401.40 \\
& $\alpha<\beta$ & 0.87 & 1.32 & 1.32 & 575.69 & 396.63 \\
& $\alpha=\beta$ & 0.88 & 1.33 & 1.33 & 576.93 & 397.96 \\
0.06 & $\alpha>\beta$ & 0.89 & 1.33 & 1.33 & 578.19 & 394.50 \\
& $\alpha<\beta$ & 0.88 & 1.16 & 1.35 & 580.333 & 395.79 \\
\hline \multirow{3}{*}{0.08} & $\alpha=\beta$ & 0.89 & 1.17 & 1.36 & 581.61 & 397.04 \\
& $\alpha>\beta$ & 0.89 & 1.17 & 1.36 & 582.89 & 392.35 \\
\hline
\end{tabular}

Table 2

Effect of non deteriorating period $\left(t_{d}\right)$ on optimal solution

\begin{tabular}{cccccccr}
\hline$t_{d}$ & $t_{r}$ & $t_{w}$ & $T$ & $Q$ & Deterioration cost & $\begin{array}{r}\text { Present Worth of Total } \\
\text { Optimal Cost }\end{array}$ & Feasible Case \\
\hline 0 & 1 & 1.28 & 1.45 & 553.46 & 72.88 & 389.35 & Case1 \\
0.08 & 0.91 & 1.18 & 1.35 & 584.19 & 35.49 & 398.24 & Case1 \\
0.25 & 0.90 & 1.18 & 1.34 & 581.95 & 24.23 & 383.60 & Case 1 \\
0.50 & 0.49 & 1.03 & 1.15 & 532.03 & 5.90 & 338.03 & Case2 \\
0.75 & 0.67 & 0.98 & 0.92 & 459.02 & 5.81 & 292.66 & Case2 \\
\hline
\end{tabular}

Table 3

Impact of $\delta$ on the optimal replenishment policy

\begin{tabular}{cccccc}
\hline$\delta$ & $t_{r}$ & $t_{w}$ & $T$ & $Q$ & $\begin{array}{c}\text { Present Worth of } \\
\text { Total Optimal Cost }\end{array}$ \\
\hline 1.2 & 0.91 & 1.19 & 1.34 & 577.26 & 401.41 \\
0.9 & 0.90 & 1.18 & 1.35 & 584.19 & 398.24 \\
0.6 & 0.89 & 1.16 & 1.36 & 593.06 & 394.12 \\
0.3 & 0.87 & 1.15 & 1.38 & 604.91 & 388.58 \\
\hline
\end{tabular}




\section{Managerial Implications:}

$>$ It is observed from Table 1 that for any fixed value of $\boldsymbol{R}$ (net rate of inflation), as the deterioration rate $(\boldsymbol{\alpha} \boldsymbol{o r} \boldsymbol{\beta})$ increases at either warehouse then there is significant increase in present worth of total optimal cost $(T C) \&$ decrease in order quantity $(Q)$ because a rise in deterioration rate $(\boldsymbol{\alpha}$ or $\boldsymbol{\beta})$ causes an increase in the cost of deteriorated units, which ultimately increase the total cost.

Also, for constant level of deterioration rate $(\boldsymbol{\alpha} \boldsymbol{o r} \boldsymbol{\beta})$ at either warehouse, an increase in net rate of inflation $(\boldsymbol{R})$ increases the order quantity $(Q)$ and present worth of total optimal cost $(T C)$. Analysis on $R$ shows that the influence of inflation should be considered even if it is small. In order to achieve higher sales during inflationary conditions, the retailer should order more for a longer period of time. It shows that as deterioration increases one should order less while if the inflation increases, one should order more. Hence the present analysis assists the decision maker in taking optimal decision under such non-reciprocal situations.

From Table 2 it is observed that as non deteriorating period $\left(t_{d}\right)$ increases, the deterioration cost will decrease significantly and hence the present worth of total optimal cost (TC) will also decreases, but the cycle length $(T)$ and the order quantity $(Q)$ decreases. This shows the importance of non instantaneous deterioration on the decision making of optimal policy. Further, when $t_{d}=0$ i.e. deterioration is instantaneous; we observe that the deterioration cost increases as compared to the non instantaneous deterioration case. Hence the present model of non instantaneous deteriorating items is very much useful in reducing the present worth of total cost $(T C)$.

$>$ Table 3 clearly shows that there is decrease in present worth of total optimal cost $(T C)$ and increases in optimal cycle length $(T)$ and order quantity $(Q)$ with the decrease in backlogging parameter $(\delta)$. As increasing the backlogging rate implies enhance in backlogged demand, thereby increasing the order quantity, but at the same time the initial inventory for the cycle decreases, which decreases the holding cost, in due course drop off present worth of total optimal cost $(T C)$. So it is advisable that when the backlogging rate is more, the decision maker should order a larger quantity in order to satisfy the backlogged demand.

\section{Conclusion and Future Scope}

In this study, a two-warehouse inventory model for non-instantaneous deteriorating items with deterministic demand under inflationary condition has been developed. Shortages are allowed and partially backlogged. The deterioration rate in OW is assumed to be higher than in RW, and the holding cost in RW is greater than that in OW because of the better storage facilities provided in RW. The items stored have the property of non-instantaneous deterioration. A mathematical model has been developed to determine the optimal ordering policy which minimizes the present worth of total optimal cost. The two considered phenomena viz., deterioration and inflation play opposite roles in the decision making process. While deterioration suggests one to order a smaller lot; high inflation recommends ordering more. Thus the model proves to be a decisive tool for the decision maker in such a contrasting scenario. The study concludes with numerical examples and sensitivity analysis so as to provide some important managerial implications. For future research, the present model can be formulated by considering permissible delay in payments, and two-level trade credit policy also can be incorporated. To be more realistic, the model can be extended with different types of variable demand such as stock-dependent demand, credit linked demand and advertisement dependent demand, etc.

\section{Acknowledgment}

The first author acknowledges the support of the University Grants Commission through University of Delhi (Research Grant No. RC/2015/9677). The second author would like to thank University Grant Commission (UGC) for providing the Non-NET fellowship to accomplish this research. 


\section{References}

Aggarwal, K. K., Aggarwal, S. P., \& Jaggi, C. K. (1997). Impact of inflation and credit policies on economic ordering. Bulletin of Pure and Applied Sciences, 16(1), 93-100.

Bakker, M., Riezebos, J., \& Teunter, R. H. (2012). Review of inventory systems with deterioration since 2001. European Journal of Operational Research, 221(2), 275-284.

Bose, S., Goswami, A., \& Chaudhuri, K. S. (1995). An EOQ model for deteriorating items with linear time-dependent demand rate and shortages under inflation and time discounting. Journal of the Operational Research Society, 46(6), 771-782.

Bierman, H., \& Thomas, J. (1977). Inventory decisions under inflationary conditions. Decision Sciences, 8(1), 151-155.

Bhunia, A. K., Jaggi, C. K., Sharma, A., \& Sharma, R. (2014). A two-warehouse inventory model for deteriorating items under permissible delay in payment with partial backlogging. Applied Mathematics and Computation, 232, 1125-1137.

Buzacott, J.A. (1975). Economic order quantity with inflation. Operations Research Quarterly, 26(3), $553-558$.

Chang, C. T., Teng, J. T., \& Goyal, S. K. (2010). Optimal replenishment policies for non-instantaneous deteriorating items with stock-dependent demand. International Journal of Production Economics, 123(1), 62-68.

Chung, K. J., \& Lin, C. N. (2001). Optimal inventory replenishment models for deteriorating items taking account of time discounting. Computer and Operational Research, 28, 67-83.

Covert, R. P., \& Philip, G. C. (1973). An EOQ model for items with Weibull distribution deterioration. AIIE transactions, 5(4), 323-326.

Dye, C. Y., Ouyang, L. Y., \& Hsieh, T. P. (2007). Deterministic inventory model for deteriorating items with capacity constraint and time-proportional backlogging rate. European Journal of Operational Research, 178(3), 789-807.

Dye, C. Y. (2013). The effect of preservation technology investment on a non-instantaneous deteriorating inventory model. Omega, 41(5), 872-880.

Ghare, P. M., \& Schrader, G. F. (1963). A model for exponentially decaying inventory. Journal of industrial Engineering, 14(5), 238-243.

Geetha, K. V., \& Uthayakumar, R. (2010). Economic design of an inventory policy for non-instantaneous deteriorating items under permissible delay in payments. Journal of Computational and Applied Mathematics, 233(10), 2492-2505.

Goyal, S. K., \& Giri, B. C. (2001). Recent trends in modeling of deteriorating inventory. European Journal of operational research, 134(1), 1-16.

Hartley VR (1976) Operations Research - A Managerial Emphasis. Good Year, Santa Monica, California, 315-317.

Hsieh, T. P., Dye, C. Y., \& Ouyang, L. Y. (2008). Determining optimal lot size for a two-warehouse system with deterioration and shortages using net present value. European Journal of Operational Research, 191(1), 182-192.

Jaggi, C. K., Aggarwal, K. K., \& Goel, S. K. (2006). Optimal order policy for deteriorating items with inflation induced demand. International Journal of Production Economics, 103(2), 707-714.

Jaggi, C.K. \& Khanna, A. (2009). The retailer's procurement policy with credit-linked demand under inflationary conditions. International Journal of Procurement Management, 2(2), 163-179.

Jaggi, C. K., \& Verma, P. (2010). A deterministic order level inventory model for deteriorating items with two storage facilities under FIFO dispatching policy. International Journal of Procurement Management, 3(3), 265-278.

Jaggi, C. K., \& Verma, P. (2010). An optimal replenishment policy for non-instantaneous deteriorating items with two storage facilities. International Journal of Services Operations and Informatics, 5(3), 209-230. 
Jaggi, C. K., Pareek, S., Khanna, A., \& Sharma, R. (2014). Credit financing in a two-warehouse environment for deteriorating items with price-sensitive demand and fully backlogged shortages. Applied Mathematical Modelling, 38(21-22), 5315-5333.

Lee, C. C., \& Hsu, S. L. (2009). A two-warehouse production model for deteriorating inventory items with time-dependent demands. European Journal of Operational Research, 194(3), 700-710.

Liang, Y., \& Zhou, F. (2011). A two-warehouse inventory model for deteriorating items under conditionally permissible delay in payment. Applied Mathematical Modelling, 35(5), 2221-2231.

Maihami, R., \& Kamalabadi, I. N. (2012a). Joint pricing and inventory control for non-instantaneous deteriorating items with partial backlogging and time and price dependent demand. International Journal of Production Economics, 136(1), 116-122.

Maihami, R., \& Abadi, I. N. K. (2012b). Joint control of inventory and its pricing for non-instantaneously deteriorating items under permissible delay in payments and partial backlogging. Mathematical and Computer Modelling, 55(5), 1722-1733.

Misra, R. B. (1975). Optimum production lot size model for a system with deteriorating inventory. International Journal of Production Research, 13(5), 495-505.

Misra, R. B. (1979). A note on optimal inventory management under inflation. Naval Research Logistics Quarterly, 26(1), 161-165.

Moon, I., \& Lee, S. (2000). The effects of inflation and time-value of money on an economic order quantity model with a random product life cycle. European Journal of Operational Research, 125(3), 588-601.

Niu, B., \& Xie, J. (2008). A note on "Two-warehouse inventory model with deterioration under FIFO dispatch policy". European Journal of Operational Research, 190(2), 571-577.

Ouyang, L. Y., Wu, K. S., \& Yang, C. T. (2006). A study on an inventory model for non-instantaneous deteriorating items with permissible delay in payments. Computers \& Industrial Engineering, 51(4), 637-651.

Ouyang, L. Y., Wu, K. S., \& Yang, C. T. (2008). Retailer's ordering policy for non-instantaneous deteriorating items with quantity discount, stock-dependent demand and stochastic backorder rate. Journal of the Chinese Institute of Industrial Engineers, 25(1), 62-72.

Raafat, F. (1991). Survey of literature on continuously deteriorating inventory models. Journal of the Operational Research society, 42(1): 27-37.

Sarker, B. R., Jamal, A. M. M., \& Wang, S. (2000). Supply chain models for perishable products under inflation and permissible delay in payment. Computers \& Operations Research, 27(1), 59-75.

Sarkar, B., \& Moon, I. (2011). An EPQ model with inflation in an imperfect production system. Applied Mathematics and Computation, 217(13), 6159-6167.

Shah, N. H., Soni, H. N., \& Patel, K. A. (2013). Optimizing inventory and marketing policy for noninstantaneous deteriorating items with generalized type deterioration and holding cost rates. Omega, 41(2), 421-430.

Soni, H. N., \& Patel, K. A. (2013). Joint pricing and replenishment policies for non-instantaneous deteriorating items with imprecise deterioration free time and credibility constraint. Computers \& Industrial Engineering, 66(4), 944-951.

Tsao, Y. C. (2014). Joint location, inventory, and preservation decisions for non-instantaneous deterioration items under delay in payments. International Journal of Systems Science, (ahead-of-print), $1-14$.

Wee, H. M., \& Law, S. T. (2001). Replenishment and pricing policy for deteriorating items taking into account the time-value of money. International Journal of Production Economics, 71(1), 213-220.

Wu, K. S., Ouyang, L. Y., \& Yang, C. T. (2006). An optimal replenishment policy for non-instantaneous deteriorating items with stock-dependent demand and partial backlogging. International Journal of Production Economics, 101(2), 369-384.

Wu, K. S., Ouyang, L. Y., \& Yang, C. T. (2009). Coordinating replenishment and pricing policies for noninstantaneous deteriorating items with price-sensitive demand. International Journal of Systems Science, 40(12), 1273-1281. 
Yang, H. L., Teng, J. T., \& Chern, M. S. (2001). Deterministic inventory lot-size models under inflation with shortages and deterioration for fluctuating demand. Naval Research Logistics (NRL), 48(2), 144158.

Yang, H. L. (2004). Two-warehouse inventory models for deteriorating items with shortages under inflation. European Journal of Operational Research, 157(2), 344-356.

Yang, H. L. (2006). Two-warehouse partial backlogging inventory models for deteriorating items under inflation. International Journal of Production Economics, 103(1), 362-370.

Yang, H. L. (2012). Two-warehouse partial backlogging inventory models with three-parameter Weibull distribution deterioration under inflation. International Journal of Production Economics, 138(1), 107116.

Zhou, Y. W., \& Yang, S. L. (2005). A two-warehouse inventory model for items with stock-leveldependent demand rate. International Journal of Production Economics, 95(2), 215-228.

\section{Appendix 1}

For Case1, the necessary and sufficient conditions for minimizing the total cost are given by

$$
\begin{aligned}
& \frac{\partial T C_{1}\left(t_{r}, T\right)}{\partial t_{r}}=\frac{D}{T}\left[\frac{F}{R}\left\{(D+1)\left(1-e^{-R t_{d}}\right) e^{\beta\left(t_{r}-t_{d}\right)}+\frac{1}{(\beta+R)}\left\{\beta e^{(\beta+R)\left(t_{r}-t_{d}\right)-R t_{r}}-R \mathrm{e}^{-R t_{t}}\right\}\right\}+\left(\frac{H}{\alpha}+c\right)\left\{\frac { 1 } { ( \alpha + R ) } \left\{\alpha e^{(\alpha+R)\left(t_{w}-t_{r}\right)-R t_{t_{r}}}\right.\right.\right. \\
& \left.\left.+R \mathrm{e}^{-R t_{w}}\right\} X_{1}-e^{(\alpha+R)\left(t_{w}-t_{r}\right)-R t_{r}}-e^{-R t_{t_{w}}}\left(1+X_{1}\right)+\left(e^{-R t_{r}}+e^{-R t_{w}}\right)\right\} \\
& \left.+\frac{s e^{-\delta T}}{\delta}\left\{\frac{1}{R}\left(\delta e^{\delta t_{w}-R T}-(\delta-R) e^{(\delta-R) t_{w}}\right)-e^{(\delta-R) t_{w}}\right\} X_{1}-c_{1} e^{-R T}\left\{1+e^{-\delta\left(T-t_{w}\right)}\right\} X_{1}\right]=0 \\
& \frac{\partial T C_{1}}{\partial T}=-\frac{1}{T^{2}}\left[A+F\left\{\frac{Z-W}{R}-\frac{\left(Z-W-D t_{d}\right) e^{-R t_{d}}}{R}+\frac{D}{R^{2}}\left\{e^{-R t_{d}}-1\right\}+\frac{D}{\beta}\left\{\frac{1}{\beta+R}\left\{e^{\beta t_{t}-(\beta+R) t_{d}}-e^{-R t_{t}}\right\}+\frac{1}{R}\left\{e^{-R t_{r}}-e^{-R t_{d}}\right\}\right\}\right\}\right. \\
& +H\left\{\frac{W}{R}\left\{1-e^{-R t_{d}}\right\}+\frac{D}{R+\alpha}\left\{e^{-R t_{d}}-e^{\alpha t_{t}-(R+\alpha) t_{r}}\right\}+\frac{D}{\alpha}\left\{\frac{1}{R+\alpha}\left\{e^{\alpha t_{w}-(R+\alpha) t_{t}}-e^{-R_{t_{t}}}\right\}+\frac{1}{R}\left\{e^{-R t_{t_{w}}}-e^{-R t_{t}}\right\}\right\}\right\} \\
& +\frac{s D}{\delta} e^{-\delta T}\left\{\frac{1}{\delta-R}\left\{e^{(\delta-R) T}-e^{(\delta-R) t_{w}}\right\}+\frac{e^{\delta t_{w}}}{R}\left\{e^{-R T}-e^{-R t_{w}}\right\}\right\}+c_{1} D e^{-R T}\left\{T-t_{w}-\frac{1}{\delta}\left\{1-e^{-\delta\left(T-t_{w}\right)}\right\}\right\} \\
& \left.+c D\left\{\frac{1}{\beta+R}\left\{e^{\beta t_{t}-(\beta+R) t_{t_{d}}}-e^{-R_{t_{r}}}\right\}+\frac{1}{\alpha+R}\left\{e^{\alpha t_{w}-(\alpha+R) t_{r}}-e^{-R_{t_{w}}}\right\}+\frac{1}{R}\left\{e^{-R t_{t_{w}}}-e^{-R_{t_{r}}}\right\}\right\}\right] \\
& +\frac{1}{T}\left[-s D e^{-\delta T}\left\{\frac{1}{\delta-R}\left\{e^{(\delta-R) T}-e^{(\delta-R) t_{w}}\right\}+\frac{e^{\delta t_{w}}}{R}\left\{e^{-R T}-e^{-R t_{w}}\right\}\right\}+\frac{s D}{\delta} e^{-\delta T}\left\{e^{(\delta-R) T}-e^{\delta t_{w}-R T}\right\}\right. \\
& \left.+c_{1} D e^{-R T}\left\{1-e^{-\delta\left(T-t_{w}\right)}\right\}-c_{1} D R \mathrm{e}^{-R T}\left\{T-t_{w}-\frac{1}{\delta}\left\{1-e^{-\delta\left(T-t_{w}\right)}\right\}\right\}\right]=0
\end{aligned}
$$

For Case1, the sufficient conditions for minimizing the total cost are given by

$$
\begin{aligned}
\frac{\partial^{2} T C_{1}}{\partial t_{r}^{2}}= & \frac{D}{T}\left[\frac{F}{R}\left\{\beta(D+1)\left(1-e^{-R t_{d}}\right) e^{\beta\left(t_{r}-t_{d}\right)}+\frac{\beta^{2}}{(\beta+R)} e^{(\beta+R)\left(t_{r}-t_{d}\right)-R t_{r}}+\frac{R^{2}}{(\beta+R)} \mathrm{e}^{-R t_{r}}\right\}+\left(\frac{H}{\alpha}+c\right)\right. \\
& \left\{\left\{\frac{1}{(\alpha+R)}\left\{\alpha e^{(\alpha+R)\left(t_{w}-t_{r}\right)-R t_{w}}+\operatorname{Re}^{-R t_{w}}\right\} X_{2}+\left\{\frac{\alpha e^{(\alpha+R)\left(t_{w}-t_{r}\right)-R t_{w}}}{(\alpha+R)}\left\{(\alpha+R)\left(X_{1}-1\right)-R X_{1}\right\}-\frac{R^{2}}{(\alpha+R)} e^{-R t_{t_{w}}} X_{1}\right\} X_{1}\right.\right. \\
& \left.-e^{(\alpha+R)\left(t_{w}-t_{r}\right)-R t_{r}}\left\{(\alpha+R)\left(X_{1}-1\right)-R\right\}+R e^{-R t_{w}} \frac{\partial t_{w}}{\partial t_{r}}\left(1+X_{1}\right)-e^{-R t_{w}} \frac{\partial^{2} t_{w}}{\partial t_{r}^{2}}-R\left(e^{-R t_{r}}+e^{-R t_{w}} X_{1}\right)\right\} \\
& +\frac{s e^{-\delta T}}{\delta}\left\{\frac{1}{R}\left(\delta^{2} e^{\delta t_{w}-R T}-(\delta-R)^{2} e^{(\delta-R) t_{w}}\right)-e^{(\delta-R) t_{w}}\right\}\left(X_{1}\right)^{2} \\
& \left.+\frac{s e^{-\delta T}}{\delta}\left\{\frac{1}{R}\left(\delta e^{\delta t_{w}-R T}-(\delta-R) e^{(\delta-R) t_{w}}\right)-e^{(\delta-R) t_{w}}\right\} X_{1}-c_{1} e^{-R T}\left\{1+e^{-\delta\left(T-t_{w}\right)}\right\} \frac{\partial^{2} t_{w}}{\partial t_{r}^{2}}+c_{1} \delta e^{-R T} e^{-\delta\left(T-t_{w}\right)}\left(X_{1}\right)^{2}\right]
\end{aligned}
$$




$$
\begin{aligned}
\frac{\partial^{2} T C_{1}}{\partial T^{2}}=\frac{2}{T^{3}}[ & A+F\left\{\frac{Z-W}{R}-\frac{\left(Z-W-D t_{d}\right) e^{-R t_{d}}}{R}+\frac{D}{R^{2}}\left\{e^{-R t_{d}}-1\right\}+\frac{D}{\beta}\left\{\frac{1}{\beta+R}\left\{e^{\beta t_{r}-(\beta+R) t_{d}}-e^{-R t_{r}}\right\}+\frac{1}{R}\left\{e^{-R t_{r}}-e^{-R t_{d}}\right\}\right\}\right\} \\
& +H\left\{\frac{W}{R}\left\{1-e^{-R t_{d}}\right\}+\frac{D}{R+\alpha}\left\{e^{-R t_{d}}-e^{\alpha t_{d}-(R+\alpha) t_{r}}\right\}+\frac{D}{\alpha}\left\{\frac{1}{R+\alpha}\left\{e^{\alpha t_{w}-(R+\alpha) t_{r}}-e^{-R t_{w}}\right\}+\frac{1}{R}\left\{e^{-R t_{w}}-e^{-R t_{r}}\right\}\right\}\right\} \\
& \left.+\frac{s D}{\delta} e^{-\delta T}\left\{\frac{1}{\delta-R}\left\{e^{(\delta-R) T}-e^{(\delta-R) t_{w}}\right\}+\frac{e^{\delta t_{w}}}{R}\left\{e^{-R T}-e^{-R t_{w}}\right\}\right\}+c_{1} D e^{-R T}\left\{T-t_{w}-\frac{1}{\delta}\left\{1-e^{-\delta\left(T-t_{w}\right)}\right\}\right\}\right] \\
& -\frac{2}{T^{2}}\left[-s D e^{-\delta T}\left\{\frac{1}{\delta-R}\left\{e^{(\delta-R) T}-e^{(\delta-R) t_{w}}\right\}+\frac{e^{\delta t_{w}}}{R}\left\{e^{-R T}-e^{-R t_{w}}\right\}\right\}+\frac{s D}{\delta} e^{-\delta T}\left\{e^{(\delta-R) T}-e^{\delta t_{w}-R T}\right\}\right. \\
& \left.+c_{1} D e^{-R T}\left\{1-e^{-\delta\left(T-t_{w}\right)}\right\}-c_{1} D R \mathrm{e}^{-R T}\left\{T-t_{w}-\frac{1}{\delta}\left\{1-e^{-\delta\left(T-t_{w}\right)}\right\}\right\}\right]+\frac{1}{T}\left[\frac{s D}{\delta} e^{-\delta T}\left\{(\delta-R) e^{(\delta-R) T}+e^{-R T}\right\}\right. \\
& -2 s D e^{-\delta T}\left\{e^{(\delta-R) T}-e^{\delta t_{w}-R T}\right\}+s D \delta e^{-\delta T}\left\{\frac{1}{\delta-R}\left\{e^{(\delta-R) T}-e^{(\delta-R) t_{w}}\right\}+\frac{e^{\delta t_{w}}}{R}\left\{e^{-R T}-e^{-R t_{w}}\right\}\right\}-2 c_{1} D R \mathrm{e}^{-R T}\left(1-e^{-\delta\left(T-t_{w}\right)}\right) \\
& \left.-c_{1} D \delta e^{-R T-\delta\left(T-t_{w}\right)}+c_{1} D R^{2} \mathrm{e}^{-R T}\left\{T-t_{w}-\frac{1}{\delta}\left\{1-e^{-\delta\left(T-t_{w}\right)}\right\}\right\}\right] \\
\frac{\partial^{2} T C_{1}}{\partial t_{r} \partial T}= & -\frac{D}{T^{2}}\left[\left(\frac{F}{\beta}+c\right) \frac{1}{(\beta+R)}\left\{\beta e^{(\beta+R)\left(t_{r}-t_{d}\right)-R t_{r}}+R \mathrm{e}^{-R t_{r}}\right\}+\left(\frac{H}{\alpha}+c\right)\left\{\frac{1}{(\alpha+R)}\left\{\alpha e^{(\alpha+R)\left(t_{w}-t_{r}\right)-R t_{w}}+R \mathrm{e}^{-R t_{w}}\right\} X_{1}\right.\right. \\
- & \left.e^{(\alpha+R)\left(t_{w}-t_{r}\right)-R t_{r}}-e^{-R t_{w}}\left(1+X_{1}\right)+\left(e^{-R t_{r}}+e^{-R t_{w}}\right)\right\}+\frac{s e^{-\delta T}}{\delta}\left\{\frac{1}{R}\left(\delta e^{\delta t_{w}-R T}-(\delta-R) e^{(\delta-R) t_{w}}\right)-e^{(\delta-R) t_{w}}\right\} X_{1} \\
- & \left.c_{1} e^{-R T}\left\{1+e^{-\delta\left(T-t_{w}\right)}\right\} X_{1}\right]+\frac{D}{T}\left[\frac{s e^{-\delta T}}{\delta}\left\{\frac{1}{R}\left(\delta e^{\delta t_{w}-R T}-(\delta-R) e^{(\delta-R) t_{w}}\right)-e^{(\delta-R) t_{w}}-\right\} X_{1}+s e^{\delta t_{w}-(\delta+R) T} X_{1}\right. \\
+ & \left.c_{1} R e^{-R T}\left\{1+e^{-\delta\left(T-t_{w}\right)}\right\} X_{1}-c_{1} \delta e^{-R T} e^{-\delta\left(T-t_{w}\right)} X_{1}\right]
\end{aligned}
$$

where $X_{1}=1-\alpha W e^{\alpha\left(t_{d}-t_{r}\right)}\left(1+\frac{\alpha W}{D} e^{\alpha\left(t_{d}-t_{r}\right)}\right) \quad$ and $\quad X_{2}=\frac{D}{D+\alpha\left(W+D\left(t_{r}-t_{d}\right)\right)}$

\section{Appendix 2}

For Case2, the necessary conditions for minimizing the total cost are given by

$$
\begin{aligned}
\frac{\partial T C_{2}}{\partial t_{r}}= & \frac{D}{T}\left[\frac{F}{R}\left(1-e^{-R t_{r}}\right)+H\left\{\frac{1}{R} e^{-R t_{d}}+\frac{1}{\alpha}\left\{\frac{1}{\alpha+R}\left\{\alpha e^{\alpha t_{w}-(\alpha+R) t_{d}}-R \mathrm{e}^{-R t_{w}}\right\}-\mathrm{e}^{-R t_{w}}\right\} Y_{1}\right\}\right. \\
+ & \frac{s e^{-\delta T}}{\delta}\left\{2 e^{(\delta-R) t_{w}}-\frac{\delta e^{-\delta t_{w}}}{R}\left(e^{-R T}-e^{-R t_{w}}\right)\right\} Y_{1}-c_{1} e^{-R T}\left(1-e^{(\delta-R)\left(T-t_{w}\right)}\right) Y_{1} \\
+ & \left.c\left\{\frac{1}{R+\alpha}\left\{\alpha e^{\alpha t_{w}-(R+\alpha) t_{d}}+R \mathrm{e}^{-R t_{w}}\right\}-\mathrm{e}^{-R t_{w}}\right\} Y_{1}\right]=0 \\
\frac{\partial T C_{2}}{\partial T}= & -\frac{1}{T^{2}}\left[A+\frac{F D}{R}\left\{t_{r}+\frac{1}{R}\left\{e^{-R t_{r}}-1\right\}\right\}+H\left\{\frac{W}{R}-\left\{W+D\left(t_{r}-t_{d}\right)\right\} \frac{e^{-R t_{d}}}{R}\right.\right. \\
& \left.+\frac{D}{\alpha}\left\{\frac{1}{\alpha+R}\left\{e^{\alpha t_{w}-(\alpha+R) t_{d}}-e^{-R t_{w}}\right\}+\frac{1}{R}\left\{e^{-R t_{w}}-e^{-R t_{d}}\right\}\right\}\right\} \\
& +\frac{s D}{\delta} e^{-\delta T}\left\{\frac{1}{\delta-R}\left\{e^{(\delta-R) t_{w}}+e^{(\delta-R) T}\right\}-\frac{e^{\delta t_{w}}}{R}\left\{e^{-R T}-e^{-R t_{w}}\right\}\right\} \\
& \left.+c_{1} D e^{-R T}\left\{T-t_{w}-\frac{1}{\delta}\left\{1-e^{-\delta\left(T-t_{w}\right)}\right\}\right\}+c D\left\{\frac{1}{R+\alpha}\left\{e^{\alpha t_{w}-(R+\alpha) t_{d}}-e^{-R t_{w}}\right\}+\frac{1}{R}\left\{e^{-R t_{w}}-e^{-R t_{d}}\right\}\right\}\right] \\
& +\frac{D}{T}\left[\frac{s}{\delta} e^{-\delta T}\left\{e^{(\delta-R) T}\right\}-e^{\delta t_{w}} \cdot e^{-R T}-s \delta e^{-\delta T}\left\{\frac{1}{\delta-R}\left\{e^{(\delta-R) t_{w}}+e^{(\delta-R) T}\right\}-\frac{e^{\delta t_{w}}}{R}\left\{e^{-R T}-e^{\left.-R t_{w}\right)}\right\}\right\}\right. \\
& c_{1} e^{-R T}\left\{1-e^{-\delta\left(T-t_{w}\right)}\right\}-c_{1} R e^{-R T}\left\{T-t_{w}-\frac{1}{\delta}\left\{1-e^{-\delta\left(T-t_{w}\right)}\right\}\right\}=0
\end{aligned}
$$


For Case 2, the sufficient conditions for minimizing the total cost are given by

$$
\begin{aligned}
& \frac{\partial^{2} T C_{2}}{\partial t_{r}^{2}}=\frac{D}{T}\left[F e^{-R t_{r}}+H\left\{\frac{1}{\alpha}\left\{\frac{1}{\alpha+R}\left\{\alpha e^{\alpha t_{w}-(\alpha+R) t_{d}}-R \mathrm{e}^{-R t_{w}}\right\}-\mathrm{e}^{-R t_{w}}\right\} Y_{2}\right\}\right. \\
& +\frac{D}{\alpha}\left\{\frac{1}{(\alpha+R)}\left\{\alpha^{2} e^{\alpha t_{w}-(\alpha+R) t_{d}}-R^{2} \mathrm{e}^{-R t_{w}}\right\}+R \mathrm{e}^{-R t_{w}}\right\}\left(Y_{1}\right)^{2}+\frac{s e^{-\delta T}}{\delta}\left\{2 e^{(\delta-R) t_{w}}-\frac{\delta e^{-\delta t_{w}}}{R}\left(e^{-R T}-e^{-R t_{w}}\right)\right\} Y_{2} \\
& +\frac{s e^{-\delta T}}{\delta}\left\{2(\delta-R) e^{(\delta-R) t_{w}}+\frac{\delta^{2} e^{-\delta t_{w}}}{R}\left(e^{-R T}-e^{-R t_{w}}\right)+\delta e^{-(\delta+R) t_{w}}\right\}\left(Y_{1}\right)^{2} \\
& -c_{1} e^{-R T}\left(1-e^{(\delta-R)\left(T-t_{w}\right)}\right) \frac{\partial^{2} t_{w}}{\partial t_{r}^{2}}-c_{1}(\delta-R) e^{-R T} e^{(\delta-R)\left(T-t_{w}\right)}\left(Y_{1}\right)^{2} \\
& \left.+c\left\{\frac{1}{R+\alpha}\left\{\alpha e^{\alpha t_{w}-(R+\alpha) t_{d}}+R \mathrm{e}^{-R t_{w}}\right\}-\mathrm{e}^{-R t_{w}}\right\}\left(Y_{1}\right)^{2}+c\left\{\frac{1}{R+\alpha}\left\{\alpha^{2} e^{\alpha t_{w}-(R+\alpha) t_{d}}+R^{2} \mathrm{e}^{-R t_{w}}\right\}+R \mathrm{e}^{-R t_{w}}\right\}\right] \\
& \frac{\partial^{2} T C_{2}}{\partial T \partial t_{r}}=\frac{D}{T}\left[\frac{F}{R}\left(1-e^{-R t_{r}}\right)+H\left\{\frac{1}{R} e^{-R t_{d}}+\frac{1}{\alpha}\left\{\frac{1}{\alpha+R}\left\{\alpha e^{\alpha t_{w}-(\alpha+R) t_{d}}-R \mathrm{e}^{-R t_{w}}\right\}-\mathrm{e}^{-R t_{w}}\right\} Y_{1}\right\}\right. \\
& +\frac{s e^{-\delta T}}{\delta}\left\{2 e^{(\delta-R) t_{w}}-\frac{\delta e^{-\delta t_{w}}}{R}\left(e^{-R T}-e^{-R t_{w}}\right)\right\} Y_{1}-c_{1} e^{-R T}\left(1-e^{(\delta-R)\left(T-t_{w}\right)}\right) Y_{1} \\
& \left.+c\left\{\frac{1}{R+\alpha}\left\{\alpha e^{\alpha t_{w}-(R+\alpha) t_{d}}+R \mathrm{e}^{-R t_{w}}\right\}-\mathrm{e}^{-R t_{w}}\right\} Y_{1}\right]+\frac{D}{T}\left[s e^{-\delta t_{w}-(\delta+R) T}-c_{1}(\delta-R) e^{(\delta-R)\left(T-t_{w}\right)-R T}\right. \\
& \left.-s e^{-\delta T}\left\{2 e^{(\delta-R) t_{w}}-\frac{\delta e^{-\delta t_{w}}}{R}\left(e^{-R T}-e^{-R t_{w}}\right)\right\}+c_{1} R e^{-R T}\left(1-e^{(\delta-R)\left(T-t_{w}\right)}\right)\right] Y_{1} \\
& \frac{\partial^{2} T C_{2}}{\partial T^{2}}=\frac{2}{T^{3}}\left[A+\frac{F D}{R}\left\{t_{r}+\frac{1}{R}\left\{e^{-R t_{r}}-1\right\}\right\}+H\left\{\frac{W}{R}-\left\{W+D\left(t_{r}-t_{d}\right)\right\} \frac{e^{-R t_{d}}}{R}\right.\right. \\
& \left.+\frac{D}{\alpha}\left\{\frac{1}{\alpha+R}\left\{e^{\alpha t_{w}-(\alpha+R) t_{d}}-e^{-R t_{w}}\right\}+\frac{1}{R}\left\{e^{-R t_{w}}-e^{-R t_{d}}\right\}\right\}\right\} \\
& +\frac{s D}{\delta} e^{-\delta T}\left\{\frac{1}{\delta-R}\left\{e^{(\delta-R) t_{w}}+e^{(\delta-R) T}\right\}-\frac{e^{\delta t_{w}}}{R}\left\{e^{-R T}-e^{-R t_{w}}\right\}\right\} \\
& \left.+c_{1} D e^{-R T}\left\{T-t_{w}-\frac{1}{\delta}\left\{1-e^{-\delta\left(T-t_{w}\right)}\right\}\right\}+c D\left\{\frac{1}{R+\alpha}\left\{e^{\alpha t_{w}-(R+\alpha) t_{d}}-e^{-R t_{w}}\right\}+\frac{1}{R}\left\{e^{-R t_{w}}-e^{-R t_{d}}\right\}\right\}\right] \\
& -\frac{2 D}{T^{2}}\left[\frac{s}{\delta} e^{-\delta T} e^{(\delta-R) T}-e^{\delta t_{w}-R T}-s \delta e^{-\delta T}\left\{\frac{1}{\delta-R}\left\{e^{(\delta-R) t_{w}}+e^{(\delta-R) T}\right\}-\frac{e^{\delta t_{w}}}{R}\left\{e^{-R T}-e^{-R t_{w}}\right\}\right\}\right. \\
& \left.+c_{1} e^{-R T}\left\{1-e^{-\delta\left(T-t_{w}\right)}\right\}-c_{1} \operatorname{Re}^{-R T}\left\{T-t_{w}-\frac{1}{\delta}\left\{1-e^{-\delta\left(T-t_{w}\right)}\right\}\right\}\right]+\frac{D}{T}\left[\frac{s}{\delta} e^{-\delta T}(\delta-R) e^{(\delta-R) T}\right. \\
& -s e^{-\delta T} e^{(\delta-R) T}+\mathrm{Re}^{\delta t_{w}-R T}+s \delta^{2} e^{-\delta T}\left\{\frac{1}{\delta-R}\left\{e^{(\delta-R) t_{w}}+e^{(\delta-R) T}\right\}-\frac{e^{\delta t_{w}}}{R}\left\{e^{-R T}-e^{-R t_{w}}\right\}\right\} \\
& -s \delta e^{-\delta T}\left\{e^{(\delta-R) T}-e^{\delta t_{w}-R T}\right\}-c_{1} \operatorname{Re}^{-R T}\left\{1-e^{-\delta\left(T-t_{w}\right)}\right\} c_{1} \delta e^{-R T-\delta\left(T-t_{w}\right)} \\
& \left.+c_{1} R^{2} e^{-R T}\left\{T-t_{w}-\frac{1}{\delta}\left\{1-e^{-\delta\left(T-t_{w}\right)}\right\}\right\}-c_{1} \operatorname{Re}^{-R T}\left\{1-e^{-\delta\left(T-t_{w}\right)}\right\}\right]
\end{aligned}
$$

where $Y_{1}=\alpha^{2} W e^{\alpha\left(t_{d}-t_{r}\right)}\left(1+\frac{2 \alpha W}{D} e^{\alpha\left(t_{d}-t_{r}\right)}\right)$ and $Y_{2}=\frac{-D^{2}}{\left\{D+\alpha\left(W+D\left(t_{r}-t_{d}\right)\right)\right\}^{2}}$ 